\title{
Seven-fluorochrome mouse M-FISH for high-resolution analysis of interchromosomal rearrangements
}

\author{
I. Jentsch, ${ }^{a, b}$ J. Geigl, ${ }^{c}$ C.A. Klein, ${ }^{c}$ and M.R. Speicher ${ }^{a, b}$ \\ a Institut für Humangenetik, Technische Universität München, München (Germany); \\ ${ }^{\mathrm{b}}$ Institut für Humangenetik, GSF-Forschungszentrum für Umwelt und Gesundheit, Neuherberg (Germany); \\ c Institut für Immunologie, Ludwig-Maximilians-Universität München, München (Germany)
}

\begin{abstract}
The mouse has evolved to be the primary mammalian genetic model organism. Important applications include the modeling of human cancer and cloning experiments. In both settings, a detailed analysis of the mouse genome is essential. Multicolor karyotyping technologies have emerged to be invaluable tools for the identification of mouse chromosomes and for the deciphering of complex rearrangements. With the increasing use of these multicolor technologies resolution limits are critical. However, the traditionally used probe
\end{abstract}

sets, which employ 5 different fluorochromes, have significant limitations. Here, we introduce an improved labeling strategy. Using 7 fluorochromes we increased the sensitivity for the detection of small interchromosomal rearrangements $(700 \mathrm{~kb}$ or less) to virtually $100 \%$. Our approach should be important to unravel small interchromosomal rearrangements in mouse models for DNA repair defects and chromosomal instability.

Copyright (C) 2003 S. Karger AG, Basel
Multi-color karyotyping technologies represent attractive approaches for detection and correct classification of complex or cryptic structural chromosomal rearrangements (for review see Fauth and Speicher, 2001). Commonly used technologies include multiplex-FISH (M-FISH, Speicher et al., 1996) or spectral karyotyping (SKY, Schröck et al., 1996). As karyotyping of mouse chromosomes is a skillful art, which is laborious work even for experienced cytogeneticists, both technologies have been extended to the analysis of mouse chromosomes (Liyanage et al., 1996; Jentsch et al., 2001). These multicolor mouse karyotyping technologies are invaluable tools as there is a growing number of mouse models for human diseases (reviewed by Resor et al., 2001). Especially important are genetically defined mouse models for the study of processes that may lead to cancer in humans (e.g. Artandi et al., 2000) and mouse

This work was supported from the Bundesministerium für Bildung und Forschung (BMBF, NGFN KB PO6T5, PO6T6). I. Jentsch received a stipend from TILL I.D., Gräfeling, Germany.

Received 9 July 2003; accepted 29 July 2003.

Request reprints from: Dr. Michael R. Speicher, Institut für Humangenetik

Technische Universität München, Trogerstr. 32, D-81675 München (Germany) telephone: +49-89-4140 6381; fax: +49-89-4140 6382

e-mail: speicher@humangenetik.med.tu-muenchen.de cloning experiments (Eggan et al., 2002). Many of these studies are associated with chromosomal aberrations and a detailed high-resolution chromosome analysis is an important cornerstone of the analyses.

However, for the karyotyping of human chromosomes we recently showed that the promises for resolution of multicolor technologies are not fulfilled with the commonly used 5-fluorochrome probe mixes (Azofeifa et al., 2000). We demonstrated that this failure is caused by the design of the probe mix. For improvement of resolution we proposed an increase of fluorochromes used for probe labeling from 5 to 7 (Azofeifa et al., 2000). This concept is outlined in Fig. 1 and its legend. Because these considerations are equally relevant for the karyotyping of murine chromosomes, we developed a 7-fluorochrome mouse M-FISH probe mix and applied it to complexly rearranged mouse chromosomes.

\section{Materials and methods}

Preparation of mouse painting probes and of fluorochrome DNA pools The generation of mouse chromosome-specific painting probes (Rabbitts et al., 1995) and their amplification by "primary" and "secondary DOPPCR" was described previously (Jentsch et al., 2001).

Similar to our human 7-Fluor M-FISH approach (Azofeifa et al., 2000) we generated a DNA pool for each fluorochrome according to the labeling

\footnotetext{
KARGER Fax +41613061234 E-mail karger@karger.ch www. karger.com

두 2003 S. Karger AG, Basel 0301-0171/03/1032-0084\$19.50/0
} 


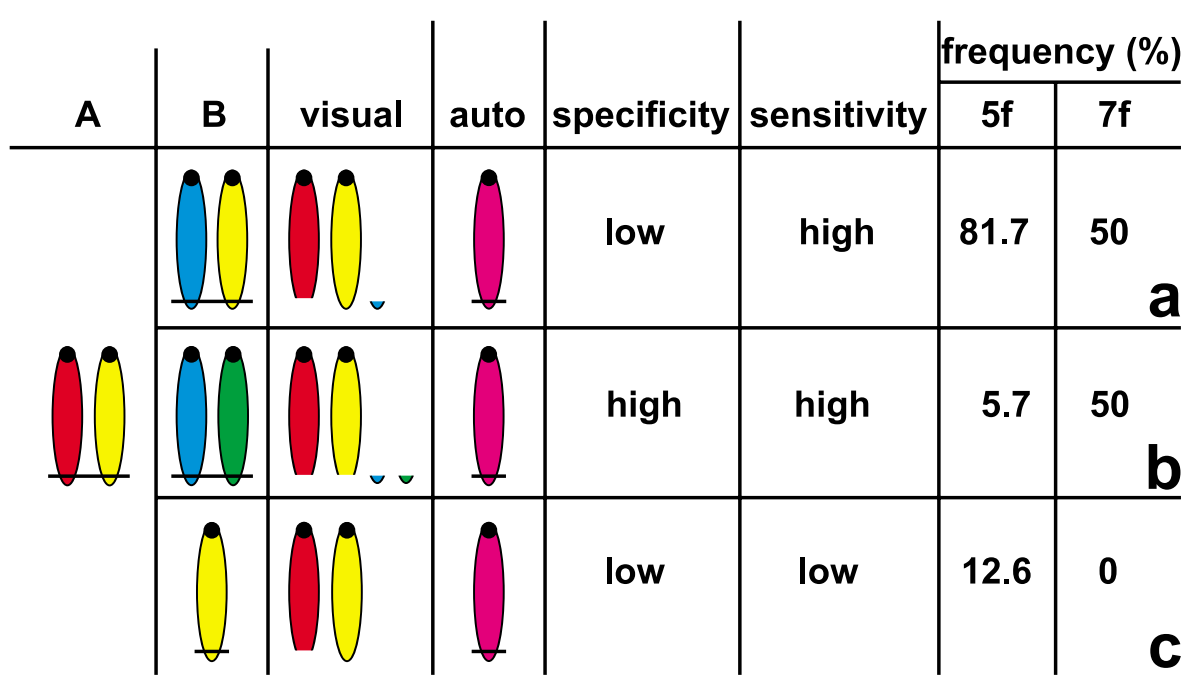

Fig. 1 . In 21-color mouse karyotyping the sensitivity and specificity for the identification of small ( $\sim 3 \mathrm{Mb}$ or less) interchromosomal rearrangements critically depends on the color-combination on derivative chromosomes. It is shown exemplarily for translocations, but the same considerations apply to small insertions as well. Translocations between chromosomes A and B are depicted. In each case chromosome A is labeled with two fluorochromes (red and yellow), while the second translocation chromosome is labeled with other fluorochrome combinations. M-FISH offers the opportunity to check hybridization results by visual inspection of individual color channels (depicted in the column "visual"). In addition, M-FISH provides an automated classification based on the spectral signature of the chromosomes (shown in the column "auto"). For simplicity only one translocation chromosome is discussed. Small interchromosomal rearrangements are identified with different sensitivity and specificity as indicated in the respective columns. The frequency of the color combinations depends critically on the number of fluorochromes and is shown for the traditionally used 5-fluorochrome mix (5f) and for our newly developed 7-fluorochrome (7f) mix. Three different types of color combinations are shown schematically for small translocations. (a, b) Small translocations are in general easily identified if the translocated segment adds an extra fluorochrome to the derivative chromosome. In (a) the two chromosomes involved in the translocation have one fluorochrome in common (yellow). The translocation will be identified with high sensitivity but low specificity because fluorescence flaring may hamper to distinguish between the possible double combinations blue/yellow or blue/ red. The frequencies for these high sensitivity/low specificity translocations are $81.7 \%$ (5 fluorochrome mix) and $50.0 \%$ (7 fluorochrome mix), respectively. (b) shows an example in which both the translocated and the centric segment are labeled each with two different fluorochromes. These translocations will be identified in any case unequivocally and have therefore both a high specificity and a high sensitivity. Such translocations are rare if a 5fluorochrome mix is used (5.7\%), but represent $50.0 \%$ of all possible translocations with the 7-fluorochrome mix. (c) Illustration of a small translocation, which has both a low sensitivity and a low specificity. Chromosome B is labeled only with the yellow fluorochrome. If part of chromosome B is translocated to chromosome A, the translocated segment is only labeled with a fluorochrome that also occurs in the centric segment. Such a small translocation may be completely obscured by fluorescence flaring and hence escape both, the visual inspection of individual fluorochrome channels (visual) and the automated classification (auto). Thus, a small interchromosomal rearrangement may be overlooked simply because of the fluorochrome composition in rearranged chromosomes. With a 5-fluorochrome 21-color-painting mix, $12.6 \%$ may be overlooked for this reason (5f). Using our optimized 7fluorochrome probe set this situation is completely avoided (for details see Azofeifa et al., 2000 and text).

\begin{tabular}{|l|c|c|c|c|c|c|c|c|c|c|c|c|c|c|c|c|c|c|c|c|c|}
\hline & 1 & 2 & 3 & 4 & 5 & 6 & 7 & 8 & 9 & 10 & 11 & 12 & 13 & 14 & 15 & 16 & 17 & 18 & 19 & $\mathrm{X}$ & $\mathrm{Y}$ \\
\hline Colour & & & & & & & & & & & & & & & & & & & & & \\
\hline DEAC & & & $\mathrm{X}$ & & $\mathrm{X}$ & & & & & $\mathrm{X}$ & $\mathrm{X}$ & & & $\mathrm{X}$ & & & & & & & $\mathrm{X}$ \\
\hline FITC & & & $\mathrm{X}$ & & & & & $\mathrm{X}$ & & & & & $\mathrm{X}$ & & $\mathrm{X}$ & & & & $\mathrm{X}$ & $\mathrm{X}$ & \\
\hline Cy3 & & & & $\mathrm{X}$ & & & & & $\mathrm{X}$ & $\mathrm{X}$ & & $\mathrm{X}$ & & & & $\mathrm{X}$ & & & $\mathrm{X}$ & & \\
\hline Cy3.5 & $\mathrm{X}$ & $\mathrm{X}$ & & & & $\mathrm{X}$ & & & & & & $\mathrm{X}$ & $\mathrm{X}$ & & & & & & & & $\mathrm{X}$ \\
\hline Cy5 & $\mathrm{X}$ & & & $\mathrm{X}$ & $\mathrm{X}$ & & & & & & & & & & & & $\mathrm{X}$ & $\mathrm{X}$ & & $\mathrm{X}$ & \\
\hline Cy5.5 & & & & & & $\mathrm{X}$ & $\mathrm{X}$ & $\mathrm{X}$ & $\mathrm{X}$ & & $\mathrm{X}$ & & & & & & & $\mathrm{X}$ & & & \\
\hline Cy7 & & $\mathrm{X}$ & & & & & $\mathrm{X}$ & & & & & & & $\mathrm{X}$ & $\mathrm{X}$ & $\mathrm{X}$ & $\mathrm{X}$ & & & & \\
\hline
\end{tabular}

Fig. 2. Labeling scheme with the color combination for 7-fluorochrome mouse M-FISH. The first row shows the chromosome numbers from 1 to 19 and the two sex chromosomes $\mathrm{X}$ and $\mathrm{Y}$. The second row depicts the pseudocolor look-up table. Rows 3-9 show the fluorochromes and the color combinations used in the experiments. Each chromosome is labeled exactly with two different fluorochromes. For example, chromosome 1 is labeled with $\mathrm{Cy} 3.5$ and $\mathrm{Cy} 5$, chromosome 2 with $\mathrm{Cy} 3.5$ and $\mathrm{Cy} 7$, and so on.

scheme shown in Fig. 2. For example, the DEAC-pool consisted of painting probes for chromosomes 3, 5, 10, 11, 14, and Y; the FITC pool of chromosomes $3,8,13,15,19$, and $\mathrm{X}$, and so on. Each painting probe was carefully calibrated to have the same fluorescent intensity after FISH according to our previously described protocols (Eils et al., 1998). After PCR labeling, the length of the DNA was adjusted by DNase I digestion to a size of 300$700 \mathrm{bp}$.

\section{Preparation of the mouse M-FISH hybridization mix}

The hybridization mix was prepared by overnight ethanol precipitation of $6 \mu \mathrm{l}$ of the DEAC-, $7 \mu \mathrm{l}$ of the FITC-, $6 \mu \mathrm{l}$ of the Cy3-, $2.5 \mu 1$ of the Texas Red(= Cy3.5), $6 \mu \mathrm{l}$ of the Cy5-, $2.5 \mu \mathrm{l}$ of the biotin- (= Cy5.5) and $3 \mu \mathrm{l}$ of the digoxigenin- (= Cy7) labeled PCR product, together with $60 \mu \mathrm{l}$ of mouse Cot-1 DNA and $20 \mu \mathrm{g}$ salmon sperm DNA. After centrifugation at 13,000 rpm for $30 \mathrm{~min}$ at $4{ }^{\circ} \mathrm{C}$ followed by a washing step with $70 \%$ ethanol, the pellet was 
resuspended in the hybridization mix, consisting of $50 \%$ formamide, $20 \%$ dextran sulfate, and $2 \times$ SSC.

\section{Induction of chromosomal aberrations and preparation of mouse}

chromosomes

The mouse chromosome preparations from mouse cell lines AG12 TUBO and TSA were done as described previously (Weaver et al., 1999) with some modifications. In brief, the spleen was isolated from mouse and transported in a tissue culture flask with RPMI/FBS. After homogenization with RPMI, the cells were incubated in a culture flask with RPMI, FBS, concanavalin A, LPS, $\beta$-mercaptoethanol. After $48 \mathrm{~h}$ incubation, the cells were treated with colcemid and $0.075 \mathrm{M} \mathrm{KCl}$ and fixed in methanol/acetic acid.

Hybridization, post-hybridization washes and detection of indirectly labeled probes

The probe mixture was denatured for $7 \mathrm{~min}$ at $78^{\circ} \mathrm{C}$ and then preannealed for about $20 \mathrm{~min}$ at $37^{\circ} \mathrm{C}$. The slides were denatured in $70 \%$ formamide, $2 \times \mathrm{SSC}$ for about $2.5 \mathrm{~min}$ at $72^{\circ} \mathrm{C}$. After passage through an ethanol series on ice, the slides were air-dried and the hybridization mixture was added to the slide. The hybridization field was sealed with a cover slip and rubber cement, and the slides were incubated for two nights at $37^{\circ} \mathrm{C}$.

Following hybridization, the slides were washed in $4 \times \mathrm{SSC} / \mathrm{T}$ ween three times $5 \mathrm{~min}$ each at $42^{\circ} \mathrm{C}$ to remove the cover slip and then three times $\left(5 \mathrm{~min}\right.$ each) with $1 \times \mathrm{SSC}$ at $60^{\circ} \mathrm{C}$. The slides were blocked in $3 \% \mathrm{BSA}$ in $4 \times$ $\mathrm{SSC} / \mathrm{T}$ ween and incubated for $30 \mathrm{~min}$ at $37^{\circ} \mathrm{C}$. After blocking, avidin Cy5.5 $(1: 100$ in $4 \% \mathrm{SSC} /$ Tween plus $1 \% \mathrm{BSA})$ and anti-digoxigenin-Cy7 (1:50 in $4 \times \mathrm{SSC} /$ Tween plus $1 \% \mathrm{BSA}$ ) were added to the slides and incubated for at least $45 \mathrm{~min}$ in a moist chamber at $37^{\circ} \mathrm{C}$. The slides were then washed three times ( 5 min each) in $4 \times \mathrm{SSC} / \mathrm{Tw}$ een at $45^{\circ} \mathrm{C}$, counterstained with DAPI (4',6-diamidino-2-phenylindole) and mounted in p-phenylenediamine dihydrochloride antifade solution.

\section{Epifluorescence microscopy}

Slides were visualized using a Leica DMRXA-RF8 epifluorescence microscope equipped with special filter blocks (Chroma Technology, Brattleboro, VT) as described (Eils et al., 1998). For image acquisition, a Sensys CCD camera (Photometrics) with a Kodak KAF 1400 chip was used. Both the camera and microscope were controlled with Leica Q-FISH software (Leica Microsystems Imaging Solutions, Cambridge, UK). Images were analyzed using the Leica MCK-Software package (Leica Microsystems Imaging Solutions, Cambridge, UK/Eils et al., 1998).

\section{Results and discussion}

\section{Testing 7-fluorochrome mouse M-FISH on normal mouse chromosomes}

The newly generated mouse M-FISH mix was tested on normal mouse metaphase spreads. The analysis of a normal female mouse metaphase spread by 7-fluorochrome mouse M-FISH is illustrated in Fig. 3. All individual color channels are shown without any image manipulation such as background subtraction or contrast enhancement. This illustrates that the spectral contrast of the epifluorescent filter cubes allowed the discrimination of adjacent fluorochromes despite significant overlap in the excitation and emission spectra. As expected, all chromosomes were classified correctly.

\section{Analysis of chromosomal aberrations with 7-fluorochrome mouse M-FISH}

We used the cell lines AG12 TUBO derived from a mammary tumor of a mouse transgenic for the Her-2/neu oncogene and TSA which was established from a moderately differentiated mammary carcinoma that spontaneously arose in a Balb/ c mouse (Rovero et al., 2000). Both cell lines revealed a number of structural and numerical chromosomal aberrations; exemplary karyotypes are shown in Fig. 4.

Cell line AG12 TUBO was diploid and the karyotype was determined as 40,XX,del(3), der(4)t(4;13),t(4;10), del(7), del(14), $-15,+\operatorname{del}(17), \operatorname{der}(17) t(15 ; 17)$ (Fig. 4A).

In the triploid cell line TSA the karyotype was deduced to be $63, \mathrm{X},-\mathrm{X},-\mathrm{X},+1,+\operatorname{der}(2) \mathrm{t}(2 ; \mathrm{X}),+\operatorname{del}(3), \operatorname{del}(4), 4,+6,7, \operatorname{dic}(8)$, $\operatorname{del}(10),+12, \operatorname{der}(13) \mathrm{t}(13 ; 14),+\operatorname{del}(15),+\operatorname{del}(15),+17,-18$ (Fig. 4B).

We have shown that the use of 7 fluorochromes for the unequivocal simultaneous labeling of all mouse chromosomes is feasible. As outlined in Fig. 1 we do not increase the number of fluorochromes to achieve a reduction of probe complexity. Instead we propose a conceptual change in probe labeling to avoid the small interchromosomal rearrangements that may be missed. Fig. 1 summarizes our approach. The basic idea is to label each chromosome with the same number of fluorochromes which results in a unique double combination of fluorochromes for each chromosome (Figs. 2 and 3). This labeling scheme ensures that each interchromosomal exchange results in the addition of at least one new fluorochrome to the derivative chromosome. In particular, in cases in which the exchanged chromosomal material is small this additional fluorochrome guarantees that the rearrangement may not be obscured by fluorescence flaring (Azofeifa et al., 2000).

As an example, in a mouse male metaphase spread with 40 chromosomes there are 420 possible two-way translocations. Using a combinatorial 5-fluorochrome labeling scheme (as done by Liyanage et al., 1996 and Jentsch et al., 2001) there are at least $53(12.6 \%)$ translocations, which result in an undetectable fluorochrome combination if the translocated material is 3 $\mathrm{Mb}$ or less (as depicted in Fig. 1c). In the two cell lines used in our experiments, we did not find such a small interchromosomal rearrangement, which would have been missed with a 5-fluorochrome probe mix. However, we recently demonstrated on human metaphase spreads that small interchromosomal rearrangements may indeed be overlooked solely because of resulting fluorochrome combinations (Azofeifa et al., 2000). Therefore, it is plausible that 7-fluorochrome mouse M-FISH can achieve the same increase in resolution. This should make our mouse multicolor technology superior to other methods.

In our human multicolor karyotyping technology we used landmarks on the $\mathrm{X}$ chromosome for the assessment of resolution limits. The different labeling of the $\mathrm{X}$ and the $\mathrm{Y}$ chromosomes results in additional bands representing the first pseudoautosomal region at Xp22.3 (PAR1, size: 2.6 Mb) and the

Fig. 3. Analysis of normal female mouse chromosomes by 7-fluorochrome mouse M-FISH. Depicted are the individual color-channels without contrast enhancement or background subtraction [DEAC (A), FITC (B), Cy3 (C), Cy3.5 (D), Cy5 (E), Cy5.5 (F), Cy7 (G)], the inverted DAPI (H) and the classified karyotype (I).

Fig. 4 . Exemplary karyotypes from mouse cell line AG12 TUBO (A) and TSA (B). 


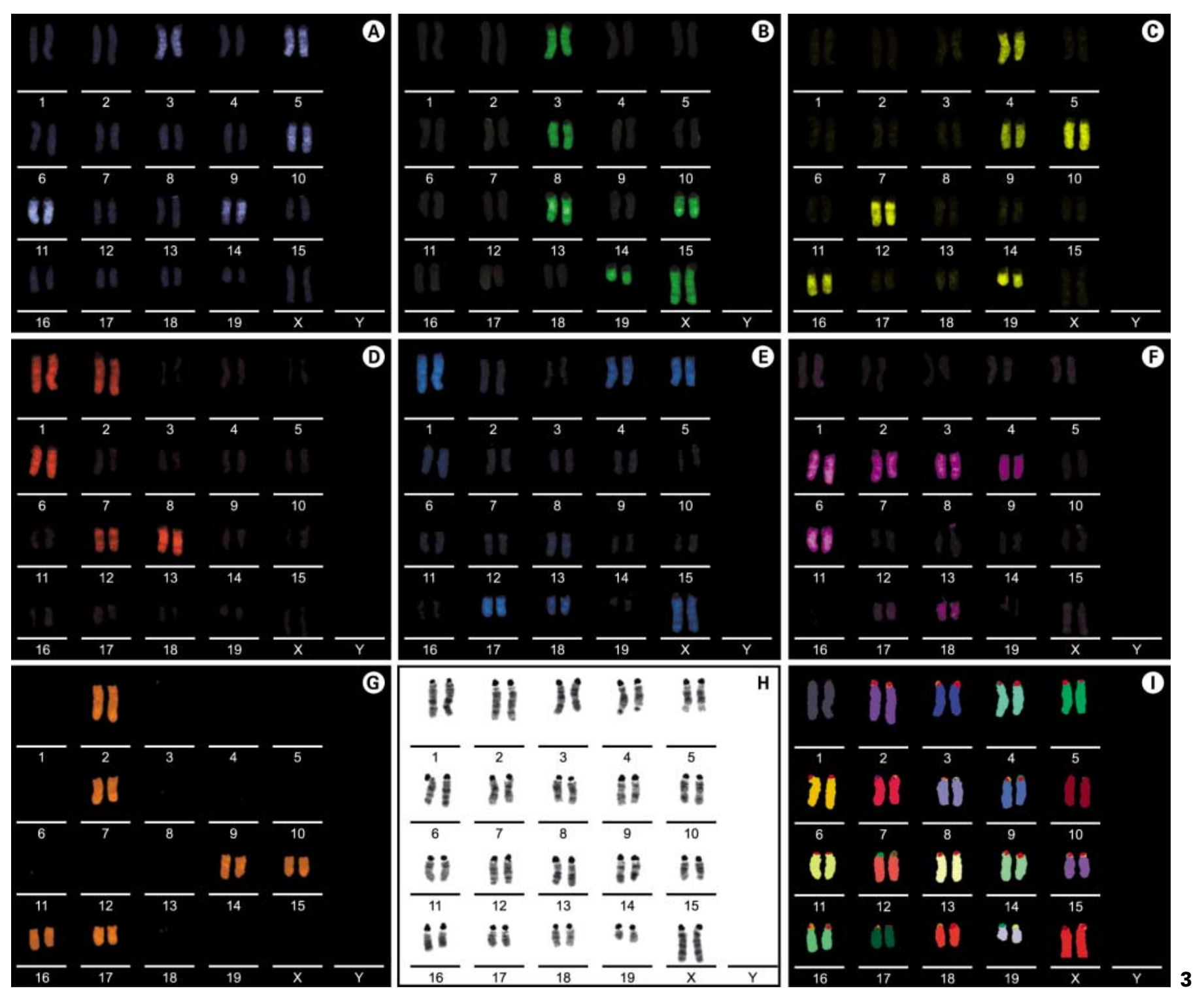

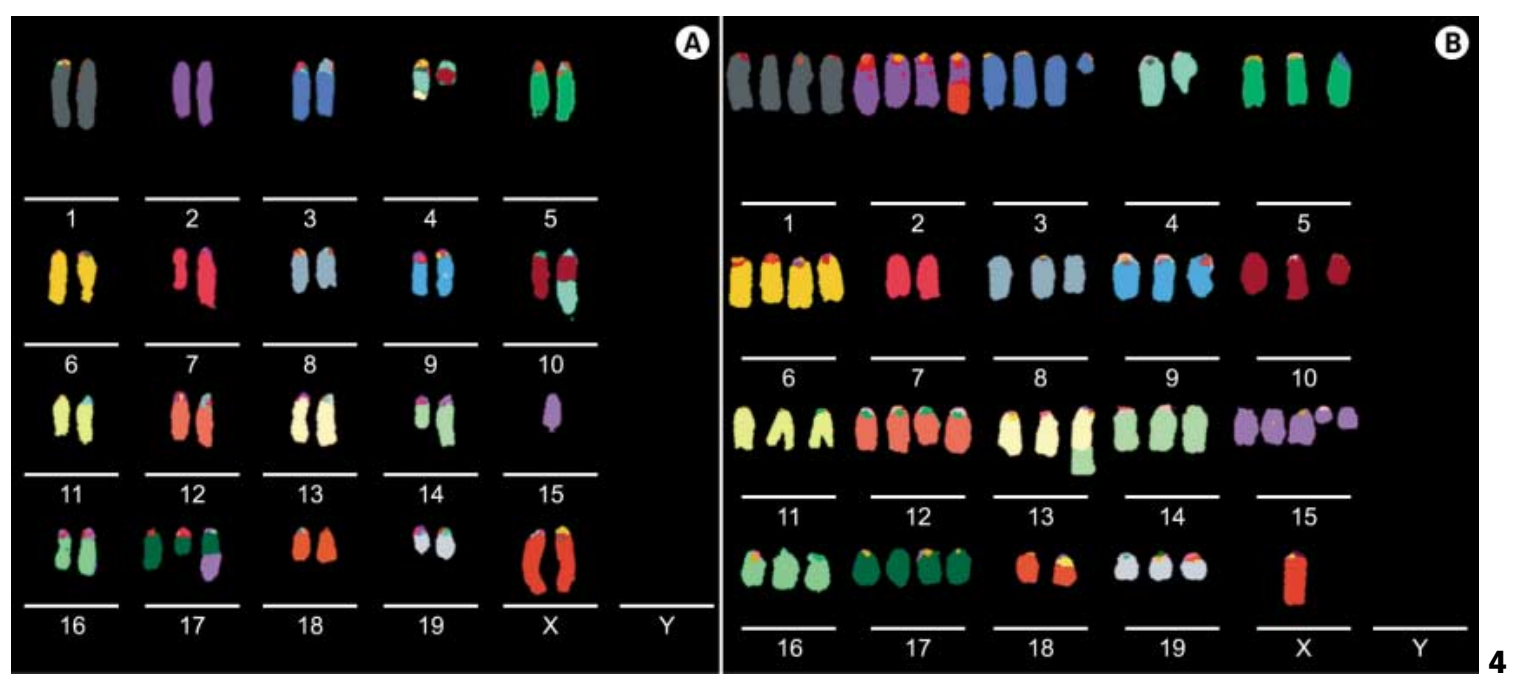


XY homology region at Xq21.3 (XY-HR, $4 \mathrm{Mb}$ ). The second pseudoautosomal region at Xq28 $(320 \mathrm{~kb})$ could never be detected (Azofeifa et al., 2000). Thus, we set the resolution limits for the detection of interchromosomal rearrangements to somewhere between $320 \mathrm{~kb}$ and $2.6 \mathrm{Mb}$. The mouse pseudoautosomal region is poorly characterized, but its size was recently estimated to be in the range of some $700 \mathrm{~kb}$ (Perry et al., 2001). We were never able to identify the mouse pseudoautosomal region. Hence, we estimate that the resolution limits in 7-fluorochrome mouse multicolor karyotyping are somewhere between $700 \mathrm{~kb}$ and $2.6 \mathrm{Mb}$.

In summary, we present an improved multicolor mouse karyotyping approach, which outperforms previously published technologies in terms of resolution. This method should be very useful for the detailed analysis of mouse genomes, which are increasingly used to model human diseases.

\section{References}

Artandi SE, Chang S, Lee SL, Alson S, Gottlieb GJ, Chin L, DePinho RA: Telomere dysfunction promotes non-reciprocal translocations and epithelial cancers in mice. Nature 406:641-645 (2000).

Azofeifa J, Fauth C, Kraus J, Speicher MR: An optimized probe set for the detection of small interchromosomal aberrations by 24-color FISH. Am J Hum Genet 66:1684-1688 (2000).

Eggan K, Rode A, Jentsch I, Samuel C, Hennek T, Tintrup H, Zevnik B, Erwin J, Loring J, Jackson-Grusby L, Speicher MR, Kuehn R, Jaenisch R: Male and female mice derived from the same embryonic stem cell clone by tetraploid embryo complementation. Nat Biotechnol 20:455-459 (2002).

Eils R, Uhrig S, Saracoglu K, Sätzler K, Bolzer A, Petersen I, Chassery JM, Ganser M, Speicher MR An optimized, fully automated system for fast and accurate identification of chromosomal rearrangements by multiplex-FISH (M-FISH). Cytogenet Cell Genet 82:160-171 (1998).

Fauth C, Speicher MR: Classifying by colors: FISHbased genome analysis. Cytogenet Cell Genet 93 . $1-10(2001)$.
Jentsch I, Adler ID, Carter NP, Speicher MR: Karyotyping mouse chromosomes by multiplex-FISH (M-FISH). Chrom Res 9:211-214 (2001).

Liyanage M, Coleman A, du Manoir S, Veldman T, McCormack S, Dickson RB, Barlow C, WynshawBoris A, Janz S, Wienberg J, Ferguson-Smith MA, Schröck E, Ried T: Multicolor spectral karyotyping of mouse chromosomes. Nature Genet 14:312-315 (1996).

Perry J, Palmer S, Gabriel A, Ashworth A: A short pseudoautosomal region in laboratory mice. Genome Res 11:1826-1832 (2001).

Rabbitts P, Impey H, Heppell-Parton A, Langford C, Tease C, Lowe N, Bailey D, Ferguson-Smith M, Carter N: Chromosome specific paints from a high resolution flow karyotype of the mouse. Nature Genet 9:369-375 (1995)

Resor L, Bowen TJ, Wynshaw-Boris A: Unraveling human cancer in the mouse: recent refinements to modeling and analysis. Hum Mol Genet 10:669675 (2001).
Rovero S, Amici A, Carlo ED, Bei R, Nanni P, Quaglino E, Porcedda P, Boggio K, Smorlesi A, Lollini PL, Landuzzi L, Colombo MP, Giovarelli M, Musiani P, Forni G: DNA vaccination against rat her2/neu p185 more effectively inhibits carcinogenesis than transplantable carcinomas in transgenic BALB/c mice. J Immunol 165:5133-5142 (2000).

Schröck E, du Manoir S, Veldman T, Schoell B, Wienberg J, Ferguson-Smith MA, Ning Y, Ledbetter DH, Bar-Am I, Soenksen D, Garini Y, Ried T (1996) Multicolor spectral karyotyping of human chromosomes. Science 273:494-497 (1996).

Speicher MR, Ballard SG, Ward DC: Karyotyping human chromosomes by combinatorial multi-fluor FISH. Nature Genet 12:368-375 (1996).

Weaver ZA, McCormack SJ, Liyanage M, du Manoir S, Coleman A, Schrock E, Dickson RB, Ried T: A recurring pattern of chromosomal aberrations in mammary gland tumors of MMTV-cmyc transgenic mice. Genes Chrom Cancer 25:251-260 (1999). 